Notch signalling is an evolutionarily conserved signalling pathway, which plays a significant role in a wide array of cellular processes including proliferation, differentiation, and apoptosis. Nevertheless, it must be noted that Notch is a binary cell fate determinant, and its overexpression has been described as oncogenic in a broad range of human malignancies. This finding led to interest in therapeutically targeting this pathway especially by the use of GSIs, which block the cleavage of Notch at the cell membrane and inhibit release of the transcriptionally active NotchIC subunit. Preclinical cancer models have clearly demonstrated that GSIs suppress the growth of such malignancies as pancreatic, breast, and lung cancer; however, GSI treatment in vivo is associated with side effects, especially those within the gastrointestinal tract. Although intensive studies are associated with the role of $\gamma$-secretase in pathological states, it should be pointed out that this complex impacts on proteolytic cleavages of around 55 membrane proteins. Therefore, it is clear that GSIs are highly non-specific and additional drugs must be designed, which will more specifically target components of the Notch signalling.

Key words: Notch signalling, cancer, GSIs.

Contemp Oncol (Pozn) 2016; 20 (4): 267-272 DOI: $10.5114 /$ wo.2016.61845

\section{Notch signalling pathway as an oncogenic factor involved in cancer development}

\author{
Marlena Brzozowa-Zasada, Adam Piecuch, Anna Dittfeld, \\ Łukasz Mielańczyk, Marek Michalski, Grzegorz Wyrobiec, \\ Marzena Harabin-Słowińska, Józef Kurek, Romuald Wojnicz
}

Department of Histology and Embryology, School of Medicine with the Division of Dentistry in Zabrze, Medical University of Silesia, Katowice, Poland

\section{Introduction}

Notch signalling is an evolutionarily conserved signalling pathway, which plays a significant role in a wide array of cellular processes including proliferation, differentiation, and apoptosis. In humans classic Notch signalling consists of four Notch receptors (NOTCH 1, 2, 3, and 4) and their ligands (DELTA-LIKE 1, 3, and 4 and JAGGED 1 and 2) [1]. All receptors are synthesised as a precursor form consisting of extracellular, transmembrane, and intracellular subunits. Within the Golgi apparatus, these precursors are cleaved by a furin-like convertase to generate two subunits. One of them contains most of the extracellular domain, and the second is composed of the rest of the extracellular and transmembrane domains. It should be mentioned that extracellular domain of the Notch receptor is composed of 36 EGF-like repeats. In the most widely accepted model of Notch activation (Fig. 1) ligand binding to EGF-like repeats unfolds the negative regulatory region (NRR) admitting the second cleavage through metalloproteinases of the ADAM family. After this, $\gamma$-secretase complex performs an intramembrane cleavage releasing the Notch intracellular domain (NotchIC or NICD) that translocates to the nucleus [2]. Importantly, for activation of Notch pathway the Mastermind-like family of proteins (MAML 1/2/3) are also needed because they form a ternary complex with CBF1-NotchIC. Then, this complex composed of CBF1-NotchIC-MAML acts as a transcriptional activator leading to Notch target gene transcription. Among the primary targets there are genes belonging to the basic helix-loop-helix (bHLH) family. Following Notch activation, the Hairy/ Enhancer-of-Split (HES) family and the Hairy-Related Transcription factor (HRT) are expressed [3].

Nevertheless, it must be noted that Notch is a binary cell fate determinant, and its overexpression has been described as oncogenic in a broad range of human malignancies $[4,5]$. This finding led to interest in therapeutically targeting this pathway especially by the use of $\gamma$-secretase inhibitors (GSIs), which block the cleavage of Notch at the cell membrane by inhibition of NotchIC release. Preclinical cancer models have clearly demonstrated that GSIs suppress the growth of such malignancies as pancreatic, breast, and lung cancer; however, GSI treatment in vivo is associated with significant side effects [6]. In this paper, we review the role of Notch signalling as an oncogenic factor involved in cancer development and the same the promising therapeutic target for GSIs activity.

\section{Aberrant Notch signalling is connected with cancer recurrence, metastasis, and treatment resistance}

Emerging evidence indicates that Notch effects are dependent on the cellular context in which it is activated (Table 1). The oncogenic potential 


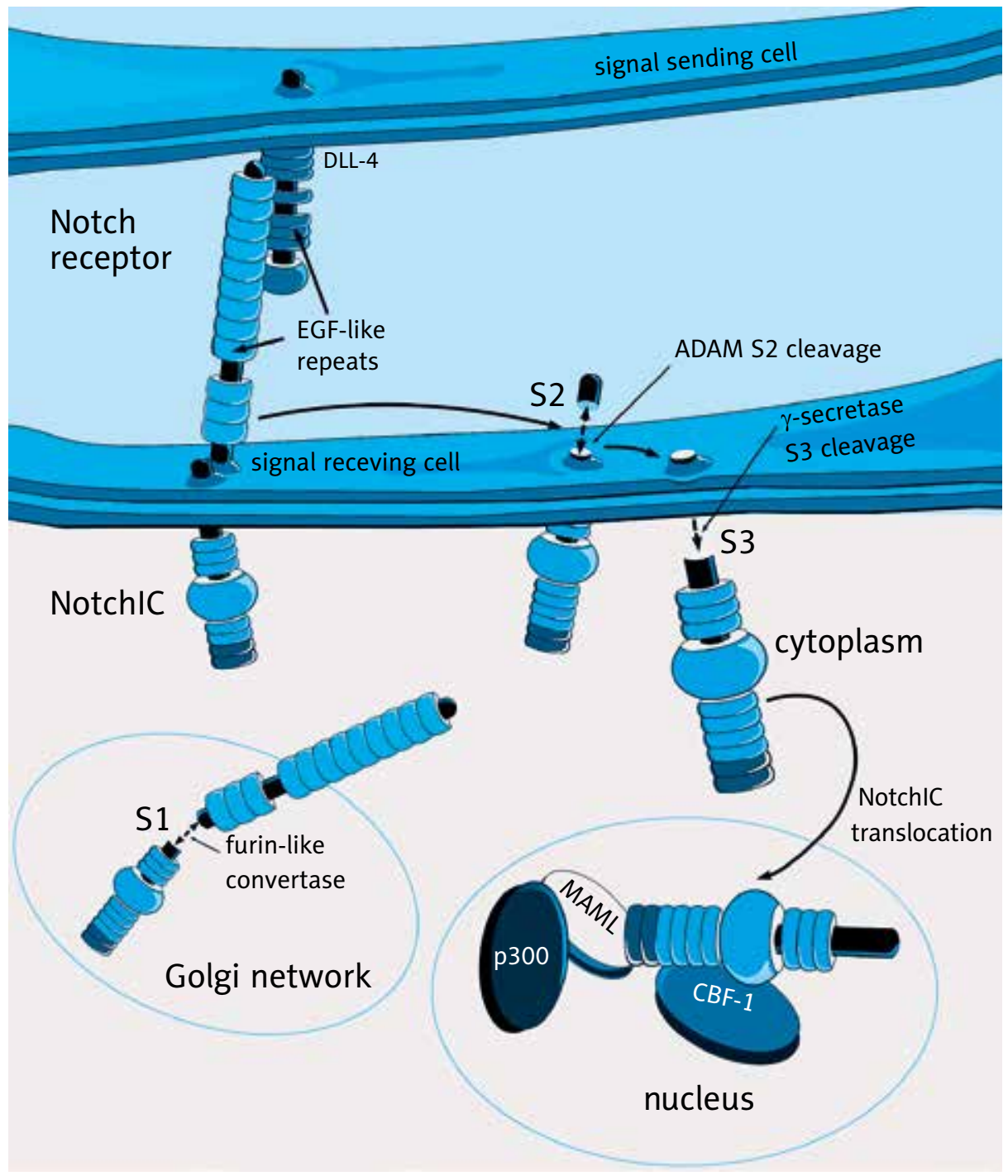

Activation of Notch signalling is associated with the interactions among a transmembrane ligand of the Delta-Serrate Lag (DSL) family and EGF-like repeats of the extracellular domain of Notch receptors. Ligand and receptor interactions are responsible for conformational changes in the receptor by exposing critical sides for ADAM17 (S2) and $\gamma$-secretase (S3) cleavage of the Notch receptor. This results in liberation and translocation of the intracellular domain (NotchIC) to the nucleus, whereas the extracellular domain (ECD) is endocytosed by the signal-sending cell. In the nucleus, NotchIC binds to CBF1, and in cooperation with Mastermind-like family of proteins (MAML 1/2/3) a ternary complex is formed. Such a ternary complex, composed of CBF1-NotchIC-MAML, acts as a transcriptional activator, leading to Notch target gene transcription. Among the primary targets there are genes belonging to the basic helix-loop-helix (bHLH) family. Following Notch activation, the Hairy/Enhancer-of-Split (HES) family and the Hairy-Related Transcription factor (HRT) are expressed.

Fig. 1. Notch signalling pathway

of the Notch pathway was first described in acute T-cell lymphoblastic leukaemia (T-ALL) in the late 1980s [7]. In recent years, Reedijk et al. revealed that high levels of JAG1 and NOTCH1 have been connected with poorer overall survival (OS) of breast cancer patients ( $p=0.01)$. It is also worth noting that a synergistic effect of these proteins on OS has been revealed as well $(p=0.003)$ [8]. Another study demonstrated that JAG1 mRNA has been associated with expression of basal breast cancer markers such as cytokeratin 5 and with reduced disease-free survival (DFS) throughout the follow-up period ( $p=0.034$ ). It has also been correlated with tumour size, oestrogen receptor status (ER), and progesterone receptor status (PgR) negativity, high tumour grade, and p53 reactivity. Moreover, tumours with high levels of both mRNA and protein demonstrated more reduced DFS in comparison to all other groups
( $p=0.020)$ [9]. Cohen et al. found that JAG1 promoted cyclin D1-mediated proliferation in the case of triple-negative breast cancers because its down-regulation was connected with inhibition of cyclin D1 expression and the same inhibition of cell cycle through the cyclin D1-dependent G1/S checkpoint [10]. Interesting results have been obtained by Simon et al., who showed that knockdown of JAG1 in mouse adrenocortical cancer cells resulted in density- dependent inhibition of cell proliferation. Co-culture experiments of normal Y1 cells with JAG1 KD or control (Scramble) cells lines demonstrated that JAG1KD cells were able to proliferate due to sufficient Jag1 signalling from adjacent cells, whereas cells receiving diminished Jag1 inputs from Jag1KD cells did not proliferate. It has been also detected in the case of cells receiving inputs from Scramble cells. Therefore, it might be said that JAG1 does not have 
a cell-autonomous effect but instead mediates cancer cell proliferation through binding to and activating Notch receptors on cells localised in the close vicinity [11]. The aberrant expression of Notch pathway is also associated with ovarian carcinoma progression. For example, the active component of the Notch1 pathway is commonly expressed in ovarian papillary serous adenocarcinoma. Depletion of NotchIC by Notch1 siRNA significantly inhibited growth of such cancer cell lines as CaOV3, OVCAR3, and SKOV3 in comparison to transfection with non-specific siRNA [12]. Ovarian cancer tissue is also characterised by the high level of Notch1 protein. Its expression enhanced gradually with the poor differentiating and increasing FIGO stage in ovarian cancer [13]. In general, the FIGO system includes five stages. Stage 0 is associated with carcinoma in situ. Stage I is associated with cancer confined to the organ of origin. Invasion of surrounding organs and tissues is related to stage II of the FIGO system. The spread to the distant nodes is a feature of stage III whereas distant metastasis is linked to stage IV [14, 15]. Overexpression of Notch proteins has been detected in the case of pancreatic adenocarcinoma. For example, NOTCH3 was shown to be overexpressed in the cytoplasm and nucleus of pancreatic cancer cells. The nuclear expression was clearly associated with poorer OS. There were also correlations between expression of NOTCH3 and such components of intracellular signalling pathways as pAkt, STAT3, and pSTAT3 [16]. This relation between overexpression of signalling molecules and poor clinical outcome may be interesting in light of findings linking Notch signalling, epigenetic silencing, and control of cell cycle during cancerogenesis [17]. Mullendore et al. revealed that pancreatic cancer cell lines are characterised by overexpression of Notch ligand transcripts such as JAG2 and DLL4 in comparison to hTERT- HPNE cells, with the majority having $>50$-fold relative expression levels. In some cells, the genomic amplification of DLL3 locus has also been observed, mirrored by overexpression of DLL3 transcripts. Interestingly, knockdown of DLL3 by RNAi was clearly connected with suppression of anchorage-independent growth in the SU86.86 cell line [18].

The aberrant Notch signalling has been involved in the EMT induction during which cancer cells acquire an invasive phenotype. This may be linked with overexpression of JAG1, NOTCH1, and consequently overexpression of Snail factors, which are known to be correlated with poor prognosis in many human cancers [19]. In this context it is worth noting study of Wang et al., who revealed that gemcitabine-resistant (GR) pancreatic cancer cells showed enhanced activation of JAG1 and NOTCH2 at the level of mRNA and protein. The GR cells transfected with NOTCH2 and JAG1 SIRNA showed increased expression of E-cadherin and reduced expression of vimentin and ZEB1. In such cases, expression of Snail1 and Snail2 have also been down-regulated. These results suggest that activation of Notch components could mediate the induction of EMT phenotype. In contrast, down-regulation of this signalling may act as a promising factor responsible for EMT inversion. What is important, down-regulation of $\mathrm{NOTCH} 2$ and JAGGED1 by siRNA transfection resulted in decreased $N F-\kappa B$ in GR cells, indicating the existence of molecular cross-talk between Notch and NF-кB [20]. Upregulation of Notch components are also associated with gastric cancer progression. For example, upregulation of DLL4 clearly promoted proliferation, migration, and invasion of SGC7901 gastric cancer cells in vitro and tumour growth in vivo. Moreover, it was also responsible for decreased activity

Table 1. Notch signalling overexpression and its role in cancer development

\begin{tabular}{|c|c|c|}
\hline Notch receptors and Notch ligands & Tumour type & Putative or detected effect \\
\hline JAG1 and NOTCH1 & Breast cancer & $\begin{array}{l}\text { Overexpression of JAG1 and NOTCH1 is associated with poorer overall } \\
\text { survival (OS) ( } p=0.01) \text {; synergistic effect of these proteins on OS has been } \\
\text { revealed, as well ( } p=0.003) \text { [8] } \\
\text { JAG1 mRNA is with expression of basal breast cancer markers such as } \\
\text { cytokeratin } 5 \text { and with reduced disease-free survival (DFS) throughout the } \\
\text { follow-up period ( } p=0.034) \text { [9] } \\
\text { Cancers with high levels of both mRNA and protein demonstrate more } \\
\text { reduced DFS in comparison to all other groups }(p=0.020) \text { [9] }\end{array}$ \\
\hline NOTCH1 & Ovarian cancer & $\begin{array}{l}\text { The active component of the Notch1 pathway is commonly expressed in } \\
\text { ovarian papillary serous adenocarcinoma [12] } \\
\text { Depletion of NotchIC by Notch1 siRNA inhibits growth of CaOV3, OVCAR3, } \\
\text { and SKOV3 cells in comparison to transfection with non-specific siRNA [12] } \\
\text { The expression of Notch1 enhances gradually with the poor differentiating } \\
\text { and increasing FIGO stage [13] }\end{array}$ \\
\hline $\mathrm{NOTCH} 3$ & $\begin{array}{l}\text { Pancreatic } \\
\text { adenocarcinoma }\end{array}$ & The nuclear expression is associated with poorer OS [14] \\
\hline DLL4 & Gastric cancer & $\begin{array}{l}\text { DLL4 overexpression promotes metastatic abilities of gastric cancer cells } \\
\text { probably by enhanced expression of MMP-9 [19] }\end{array}$ \\
\hline $\mathrm{NOTCH} 3$ & $\begin{array}{l}\text { Squamous cell } \\
\text { carcinoma }\end{array}$ & $\begin{array}{l}\text { The high level of nuclear NOTCH3 expression is associated with shorter OS } \\
\text { [20] }\end{array}$ \\
\hline NOTCH1 & Melanoma & $\begin{array}{l}\text { Constitutive activation of NOTCH1 by ectopic expression of NICD is } \\
\text { probably associated with proliferation of VGP primary melanoma cells [21] } \\
\text { NOTCH1 may promote melanoma progression by regulation of } \beta \text {-catenin } \\
\text { and cyclin D1 expression [26] }\end{array}$ \\
\hline
\end{tabular}


of MMP-9; however, its increased expression at the level of mRNA has not been detected. Probably, Notch target genes, as well as MMP-9, need post-transcriptional regulations. It is also possible that activation of MMP-9 can be suppressed by other signalling pathways or molecules activated by DLL4, e.g. tissue inhibitor of metalloproteinase-1 (TIMP-1), TIMP-2, or other TIMPs. It is also worth noting that in gastric cancer cells with overexpression of DLL4, increased level of MRNA and secretion of MMP-2 proenzymes have been observed. Probably MMP-9 signalling is not sufficient to exert an effect alone in gastric cancer progression, while other molecules such as MMP-2 play a more fundamental role [21]. As revealed by Yeasmin et al., cervical squamous cell carcinoma is also characterised by aberrant expression of Notch proteins. In contrast to cervical tissue without any pathological changes and cervical intraepithelial neoplasia, squamous cell carcinomas displayed high levels of nuclear NOTCH3 expression, which was associated with a shorter OS of such patients. Importantly, inactivation of NOTCH3 diminished cell proliferation and induced apoptosis in ME180 and SKGIllb cell lines with NOTCH3 overexpression [22]. Overexpression of Notch proteins is also associated with melanoma development. Notch1 activation may drive the vertical growth phase (VGP) of primary melanoma toward a more aggressive phenotype. Constitutive activation of NOTCH1 by ectopic expression of NotchlC was probably responsible for proliferative abilities of VGP primary melanoma cells in a serum-independent and growth factor-independent manner in vitro. NOTCH1 activation also enhanced tumour survival during culturing as three-dimensional spheroids. These effects were probably mediated by activation of the mitogen-activated protein kinase (MAPK) and Akt pathways because inhibition of MAPK or the phosphatidylinositol 3-kinase (PI3K) - Akt pathway can reverse the tumour cell growth induced by NOTCH1 [23]. Bedogni et al. demonstrated that NOTCH1 influenced melanoma development in a xenograft model by guarding cells from death [24]. Probably due to enhanced BAX/BCL-XL ratio, which in turn may be responsible for induction of caspase-3 because NOTCH1 has been shown to positively regulate $\mathrm{BCL}-\mathrm{XL}$ expression in T cells and pancreatic cancer cells, which may be responsible for resistance to apoptosis [25, 26]. Another interesting finding is associated with the observation that NOTCH1 knockdown significantly decreased cell growth due to inhibition of cyclin D1. These findings imply that inhibition of cell and tumour growth can also be dependent on reduced cyclin D1 expression [27]. NOTCH1 may promote melanoma progression by inducing $\beta$-catenin, which in turn regulate cyclin D1 expression. Nevertheless, in this system $\beta$-catenin appeared unaffected by inhibition of NOTCH1, which suggests that expression of cyclin D1 is under direct control of NOTCH1 activity [28].

\section{The most promising approach to inhibit Notch signalling is the use of $\gamma$-secretase inhibitors (GSIs)}

Proteolytic processing of Notch by $\gamma$-secretase is a necessary step in the activation of this family of proteins.
Therefore, $\gamma$-secretase protease complex has been considered as a promising target for Notch inhibition with a class of compounds called $\gamma$-secretase inhibitors (GSIs) [29]. All GSIs that impair Notch pathway act through its inhibition, despite their different chemical structures [29, 30]. Liu et al. compared the expression of Notch components in A549 cancer cells after DAPT (GSI-IX) and cisplatin (CDDP) treatment. They revealed distinctive differences in cell cycle progression. For example, some cells that display CD133 surface marker have been found in G2/M phase, and there were half as many cells in S phase in comparison to CD133negative cells. But the most important fact was associated with the observation that inhibitory effects of CDPP were enhanced when cells had been pretreated with GSI [31]. Pharmacological blockade of Notch by the use of GSIs clearly reduced the percentage of cells displaying stem cell markers. In the case of glioblastoma, cells expressing CD133 did not show the ability to form colonies in vitro or engraft in vivo [32]. Timme et al. revealed that treatment of colon cancer cells with MRK-003 reduced oxaliplatin-induced apoptotic cell death, while GSI treatment alone did not influence growth or apoptosis. Interestingly, blocking of MCL-1 and BCL-XL by siRNA or the small molecule inhibitor obatoclax restored the 'apoptotic potential' of cells treated with both oxaliplatin and MRK-003 [33]. Also, Palagni et al. revealed that dual treatment with GSI IX and AG490 clearly altered apoptosis of pancreatic ductal adenocarcinoma cells (PDAC). Moreover, proliferation, migration, and invasion of cells have also been impaired in comparison to monotherapy. It must be noted that combination treatment significantly attenuates tumour progression in vivo and inhibits transition from acinar-ductal-metaplasia to PDAC [34]. In contrast, Wang et al. demonstrated that pretreatment of ovarian cancer cells with DAPT and then with cisplatin increased apoptosis via modulation of such genes as cyclin B1, BCL-2, and caspase-3 [35]. Inhibition of NOTCH1 with sulphonamide GSI (GSI34) blunted HES1 activation in the case of colon cancer cells. After GSI34 treatment, cells showed sensitivity to chemotherapy, and this effect was synergistic with oxaliplatin, 5-FU, and SN38 [36].

Nevertheless, administration of GSIs in vivo is associated with strong side effects, especially within the gastrointestinal tract. In C57BL/ 6 and TgCRND8 APP transgenic mice, chronic administration of GSI LY-411575 was responsible for alterations in intestinal architecture, including increased goblet cell number and mucin secretion leading to epithelial erosion. Moreover, reduced overall thymic cellularity and altered intrathymic differentiation at the $\mathrm{CD} 4^{+} \mathrm{CD} 8^{-} \mathrm{CD} 44^{+} \mathrm{CD} 25^{+}$precursor stage has been observed as well [37]. It is not surprising because Hadland et al. revealed that application of GSI to foetal thymus organ cultures interferes with $T$ cell development in a manner consistent with reduction of NOTCH1 activity. It means that progression from an immature $\mathrm{CD}^{-} / \mathrm{CD}^{-}$state to an intermediate $\mathrm{CD}^{+} / \mathrm{CD}^{+}$double-positive state has been inhibited [38]. Importantly, all of these effects are consistent with the role of Notch signalling as a crucial factor responsible for developmental processes and tissue homeostasis maintaining. Although intensive studies are as- 
sociated with the role of $\gamma$-secretase in pathological states it should be pointed out that this complex influences the proteolytic cleavages of around 55 membrane proteins [39, 40]. Therefore, it is clear that GSI are highly non-specific, and additional drugs must be designed which will more specifically target Notch [41].

The majority of the trials associated with the use of GSIs in the cancer treatment are either at a stage at which it is too early to report the side effects or have been completed but these effects have not yet been published. Krop et al. showed that inhibition of Notch signalling has been detected with the 1.800-4.200 mg weekly dose levels of MK-0752. Among the most significant side effects the following should be mentioned: diarrhoea, nausea, vomiting, and fatigue [42]. In this context, it is worth noting the study of Tolcher et al., who observed such effects as fatigue, thrombocytopenia, fever, rash, chills, and anorexia. In this context it is worth noting about transient grade 3 hypophosphataemia (dose-limiting toxicity, one patient) and grade 3 pruritus (two patients), which have been reported at $27 \mathrm{mg}$ and $60 \mathrm{mg}$ of RO4929097. Furthermore, transient grade 3 asthaenia was observed in one patient at $80 \mathrm{mg}$ [43]. RO4929097 can also be safely combined with temsirolimus in patients with metastatic high-grade synovial sarcoma and in patients with metastatic gastrointestinal stromal tumours. The RP2D was established at $20 \mathrm{mg}$ of RO4929097 in combination with 37.5 mg of temsirolimus. In this case, the most common toxicities included fatigue, mucositis, neutropaenia, anaemia and hypertriglyceridaemia [44]. In patients with refractory metastatic colorectal cancer after RO4929097 administration, no radiographic responses have been detected, and time to progression was short. Median PFS was 1.8 months, and median OS was six months. Moreover, immunohistochemical analysis of cancer tissue demonstrated positive staining for the Notch receptors and NotchIC. Interestingly, the positive staining was also visible in the case of HES1 protein [45].

In summary, a great number of studies suggest a potential clinical application of GSIs in cancer therapeutics. Nevertheless, among major challenges on the way toward this goal one associated with gastrointestinal toxicity is very significant. Moreover, Notch is described to widely participate in cellular physiology, e.g. haematopoiesis and maintenance of arterial smooth muscle. So it is not surprising that inactivation of $\gamma$-secretase may result in vital organ dysfunction. Second, it is worth noting that GSIs do not exclusively target the Notch pathway because $\gamma$-secretase has many substrates including ErbB4, CD44, and syndecan-3. GSIs may also target proteases other than $\gamma$-secretase, and therefore these compounds may be responsible for adverse effects in vivo. It seems that partial inhibition of $\gamma$-secretase will be sufficient to inhibit Notch components in cancer cells whereas the dosage will not affect the functions in tissue without lesions. Nevertheless, further studies associated with GSIs are needed.

The authors declare no conflict of interest.

\section{References}

1. Fleming FJ. Structural conservation of Notch receptors and ligands. Semin Cell Dev Biol 2007; 9: 599-607.

2. Bray SJ. Notch signaling: a simply pathway become complex. Nat Rev Mol Cell Biol 2006; 7: 678-89.

3. Fryer CJ, Lamar E, Turbachova I, Kintner C, Jones KA. Mastermind mediates chromatin-specific transcription and turnover of the notch enhancer complex. Genes Dev 2002; 16: 1397-411.

4. Brzozowa M, Mielańczyk $九$, Michalski M, et al. Role of Notch signaling pathway in gastric cancer pathogenesis. Contemp Oncol (Pozn) 2013; 17: 1-5.

5. Brzozowa M, Wojnicz R, Kowalczyk-Ziomek G, Helewski K. The Notch ligand Delta-like 4 as a target in angiogenesis based cancer therapy? Contemp Oncol (Pozn) 2013; 17: 234-7.

6. Aster JC. In brief: Notch signaling in health and disease. J Pathol 2014; 232: 1-3.

7. Roma J, Masià A, Revent s J, Sánchez de Toledo J, Gallego S. Notch pathway inhibition significantly reduces rhabdomysarcoma invasiveness and mobility in vitro. Clin Cancer Res 2011; 17: 505-13.

8. Reedijk M, Odorcis S, Chang L, Zhang H, Miller N, McCready DR, Lockwood G, Egan SE. High-level coexpression of JAG1 and NOTCH1 is observed in human breast cancer and is associated with poor overall survival. Cancer Res 2005; 65: 8530-7.

9. Reedijk M, Pinnaduwage D, Dickson BC, et al. JAG1 expression is associated with a basal phenotype and recurrence in lymph node-negative breast cancer. Breast Cancer Res Treat 2008; 111: 439-48.

10. Cohen B, Shimizu M, Izrailit J, et al. Cyclin D1 is a direct target of JAG1-mediated Notch signaling in breast cancer. Breast Cancer Res Treat 2010; 123: 113-24.

11. Simon DP, Giordano TJ, Hammer GD. Upregulated JAG1 enhances cell proliferation in adrenocortical carcinoma. Clin Cancer Res 2012; 18: 2452-64.

12. Rose SL, Kunnimalaiyaan M, Drenzek J, Seiler N. Notch 1 signaling is active in ovarian cancer. Gynecol Oncol 2010; 117: 130-3.

13. Wang M, Wang J, Wang L, Wu L, Xin X. Notch1 expression correlates with tumor differentiation status in ovarian carcinoma. Med Oncol 2010; 27: 1329-35.

14. Suh DH, Kim TH, Kim JW, et al. Improvements to the FIGO staging for ovarian cancer: reconsideration of lymphatic spread and intraoperative tumor rupture. J Gynecol Oncol 2013; 24: 352-8.

15. Dai- Yuan M, Bang-Xian T, Xian-Fu L, Ye-Gin Z, Hong-Wei C. A meta analysis: neoadjuvant chemotherapy versus primary surgery in ovarian carcinoma FIGO stage III and IV. World J Surg Oncol 2013; 11: 267.

16. Doucas H, Mann CD, Sutton CD, Garcea G, Neal CB, Berry DP, Manson MM. Expression of nuclear Notch3 in pancreatic adenocarcinomas is associated with adverse clinical features and correlates with the expression of STAT3 and phosphorylated Akt. J Surg Oncol 2008; 97: 63-8.

17. Ferres-Marco D, Gutierrez-Garcia I, Vallejo DM, Bolivar J, Gutierrez-Aviño FJ, Dominguez M. Epigenetic silencer and Notch collaborate to promote malignant tumors by Rb silencing. Nature 2006; 439: 430-6.

18. Mullendore ME, Koorstra JB, Li YM, Offerhaus GJ, Fan X, Henderson CM, et al. Ligand-dependent Notch signaling is involved in tumor initiation and tumor maintenance in pancreatic cancer. Clin Cancer Res 2009; 15: 2291-301.

19. Leong KG, Niessen K, Kulic I, Raouf A, Eaves C, Pollet I, Karsan A. Jagged1-mediated Notch activation induces epithelial-to-mesenchymal transition through Slug-induced repression of E-cadherin. J Exp Med 2007; 204: 2935-48.

20. Wang Z, Li Y, Kong D, Banerjee S, et al. Acquisition of epithelial-mesenchymal transition phenotype of gemcitabine-resistant pancreatic cancer is linked with activation of the Notch signaling pathway. Cancer Res 2009; 69: 2400-7.

21. Li GG, Li L, Li C, et al. Influence of up-regulation of Notch ligand DLL4 on biological behaviors of human gastric cancer cells. World J Gastroenterol 2013; 19: 4486-94. 
22. Yeasmin S, Nakayama K, Rahman MT, et al. Expression of nuclear Notch3 in cervical squamous cell carcinoma and its association with adverse clinical outcomes. Gynecol Oncol 2010; 117: 409-16.

23. Liu ZJ, Xiao M, Balint K, et al. Notch1 signaling promotes primary melanoma progression by activating mitogen-activated protein kinase/phosphatidylinositol 3-kinase-Akt pathways and up-regulating N-cadherin expression. Cancer Res 2006; 66: 4182-90.

24. Bedogni B, Warneke JA, Nickoloff BJ, Giaccia AJ, Powell MB. Notch1 is an effector of Akt and hypoxia in melanoma development. J Clin Invest 2008; 118: 3660-70.

25. Wang Z, Zhang Y, Li Y, Banerjee S, Liao J, Sarkar FH. Down-regulation of Notch-1 contributes to cell growth inhibition and apoptosis in pancreatic cancer cells. Mol Cancer Ther 2006; 5: 483-49.

26. Bheeshmachar G, Purushotaman D, Sade H, Gunesekharan V, Rangarajan A, Sarin A. Evidence for a role for notch signaling in the cytokine-dependent survival of activated T cells. J Immunol 2006; 177: 5041-50.

27. Ronchini C, Capobianco AJ. Induction of cyclin D1 transcription and CDK2 activity by Notch(ic): implication for cell cycle disruption in transformation by Notch(ic). Mol Cell Biol 2001; 21: 5925-34.

28. Balint K, Xiao M, Pinnix CC, et al. Activation of Notch1 signaling is required for beta-catenin-mediated human primary melanoma progression. J Clin Invest 2005; 115: 3166-76.

29.Rizzo P, Osipo C, Foreman K, Golde T, Osborne B, Miele L. Rational targeting of Notch signaling in cancer. Oncogene 2008; 27: 5124 31.

30. Schott AF, Landis MD, Dontu G, et al. Preclinical and clinical studies of gamma-secretase inhibitors with docetaxel on human breast tumors. Clin Cancer Res 2013; 19: 1512-24.

31. Liu J, Mao Z, Huang J, Xie S, Liu T, Mao Z. Blocking The NOTCH pathway can inhibit the growth of CD133-positive A549 cells and sensitize to chemotherapy. Biochem Biophys Res Commun 2014; 444: 670-5

32. Fan X, Khaki L, Zhu TS, et al. NOTCH pathway blockade depletes CD133-positive glioblastoma cells and inhibits growth of tumor neurospheres and xenografts. Stem Cells 2010; 28: 5-16.

33. Timme CR, Gruidl M, Yeatman TJ. Gamma-secretase inhibition attenuates oxaliplatin-induced apoptosis through increased Mcl1 and/or Bcl-xL in human colon cancer cells. Apoptosis 2013; 18: 1163-74.

34. Palagni V, Bozko P, El Khatib M, et al. Combined inhibition of Notch and JAK/STAT is superior to monotherapies and impairs pancreatic cancer progression. Carcinogenesis 2014; 35: 859-66.

35. Wang M, Ma X, Wang J, Wang L, Wang Y. Preatreatment with the $\gamma$-secretase inhibitor DAPT sensitizes drug-resistant ovarian cancer cells to cisplatin by downregulation of Notch signaling. Int J Oncol 2014; 44: 1401-9.

36. Meng RD, Shelton CC, Li YM, et al. gamma- secretase inhibitors abrogate oxaliplatin-induced activation of the Notch-1 signaling pathway in colon cancer cells resulting in enhanced chemosensitivity. Cancer Res 2009; 69: 573-82.

37. Wong GT, Manfra D, Poulet FM, et al. Chronic treatment with the gamma-secretase inhibitor LY-411,575 inhibits beta-amyloid peptide production and alters lymphopoiesis and intestinal cell differentiation. J Biol Chem 2004; 279: 12876-12882.

38. Hadland BK, Manley NR, Su D, et al. Gamma-secretase inhibitors repress thymocyte development. Procl Natl Acad Sci USA 2001; 98: 7487-91.

39. Wolfe MS. Structure, mechanism and inhibition of gamma-secretase and presenilin-like proteases. Biol Chem 2010; 391: 839-47.

40. Li H, Wolfe MS, Selkoe DJ. Toward structural elucidation of the gamma-secretase complex. Structure 2009; 17: 326-34

41. Luistro L, He W, Smith M, et al. Preclinical Profile of a potent gamma-secretase inhibitor targeting notch signaling with in vivo ef ficacy and pharmacodynamics properties. Cancer Res 2009; 69: 7627-80.

42. Krop I, Demuth T, Guthrie T, et al. Phase I pharmacologic and pharmacodynamics study of the gamma secretase (Notch) inhibitor MK-0752 in adult patients with advanced solid tumors. J Clin Oncol 2012; 30: 2307-13

43. Tolcher AW, Messersmith WA, Mikulski SM, et al. Phase I study of RO4929097, a gamma secretase inhibitor of Notch signaling, in patients with refractory metastatic or locally advanced solid tumors. J Clin Oncol 2012; 30: 2348-53.

44. Diaz-Padilla I, Hirte H, Oza AM, et al. A phase Ib combination study of RO4929097, a gamma-secretase inhibitor, and temsirolimus in patients with advanced solid tumors. Invest New Drugs 2013; 31: 1182-91.

45. Strosberg JR, Yeatman T, Weber J, et al. A phase II of R04929097 in metastatic colorectal cancer. Eur J Cancer 2012; 48: 997-1003.

\section{Address for correspondence}

\section{Marlena Brzozowa}

Chair and Department of Histology and Embryology

Medical University of Silesia

Jordana 19

41-808 Zabrze, Poland

e-mail: marlena.brzozowa@op.pl

Submitted: 30.06 .2014

Accepted: $\quad 11.09 .2014$ 